O Genoma de Tetrapedia diversipes (Hymenoptera, Apidae) e a evolução da diapausa em abelhas. 
Priscila Karla Ferreira dos Santos

O Genoma de Tetrapedia diversipes (Hymenoptera, Apidae) e a evolução da diapausa em abelhas

The genome of Tetrapedia diversipes (Hymenoptera, Apidae) and the evolution of diapause in bees 
Priscila Karla Ferreira dos Santos

\section{O Genoma de Tetrapedia diversipes (Hymenoptera, Apidae) e a evolução da diapausa em abelhas}

\section{The genome of Tetrapedia diversipes (Hymenoptera, Apidae) and the evolution of diapause in bees}

Tese apresentada ao Instituto de Biociências da Universidade de São Paulo, para a obtenção do título de Doutora em Ciências, na área de Biologia (Genética)

Orientadora: Dra Maria Cristina Arias

São Paulo 


\section{RESUMO}

Tetrapedia diversipes é uma espécie de abelha solitária, coletora de óleo e presente na região Neotropical. Nos últimos anos tem sido nossa espécie modelo para a compreensão dos mecanismos moleculares envolvidos na diapausa. Essa espécie é bivoltina, e uma das gerações anuais passa por um período de diapausa, o que estende o tempo total de desenvolvimento. A diapausa é uma via alternativa do desenvolvimento caracterizada por mudanças fisiológicas profundas. É uma adaptação de muitos artrópodes para sobreviver às adversidades ambientais, como frio rigoroso, falta de recursos e a seca. A diapausa está presente em todas as famílias do grupo das abelhas e ocorre principalmente nos estágios de pré-pupa e adulto. Esse fenótipo, em abelhas, parece ter tido um papel fundamental na evolução do comportamento social. Uma das hipóteses aventa que o bivoltismo e a diapausa na fase adulta seriam fatores importantes para evolução do comportamento social. O Capítulo 1 desta tese é uma revisão bibliográfica das fases em que a diapausa ocorre em abelhas e de forma inovadora foi realizada uma correlação desses dados com as seguintes variáveis: tipo de ninho, voltismo, latitude e comportamento social. Nós verificamos que a fase do desenvolvimento em que a diapausa ocorre está correlacionada significativamente com essas variáveis (exceto tipo de ninho) em todas as espécies em que a socialidade surgiu. Ainda, houve perda da diapausa do desenvolvimento no grupo das abelhas sociais. Para compreender melhor os mecanismos moleculares da diapausa do desenvolvimento em $T$. diversipes, nós apresentamos no Capítulo 2 o genoma desta espécie. Nós reanalisamos os dados de transcriptoma da fase de diapausa, previamente gerados, e identificamos assinaturas genômicas relacionadas a este fenótipo, como o enriquecimento de sitíos de ligação para fatores de transcrição nas regiões promotoras dos genes diferencialmente expressos durante a diapausa. Nós também comparamos o genoma de $T$. diversipes com outros genomas de abelhas e identificamos ganhos e perdas de famílias gênicas relacionados à fase em que a diapausa ocorre. Por último, como um sub-produto do genoma de $T$. diversipes, nós apresentamos o genoma da Wolbachia endossimbionte de $T$. diversipes e uma análise populacional desta bactéria em cinco populações do hospedeiro. Nós identificamos que todos os indivíduos, das diferentes populações, eram infectados pelas mesmas linhagens de Wolbachia. Sendo assim, este endossimbionte não tem influência na estrutura populacional descrita para fêmeas, nem no viés para fêmeas ou machos descrito para algumas das populações. 


\begin{abstract}
Tetrapedia diversipes is a solitary oil-collecting bee from Neotropics. In the last years it has been our model to comprehend the molecular mechanisms involved in diapause. We chose this species because $T$. diversipes is bivoltine and the individuals from one generation have a direct development while individuals from the other generation have a longer developmental time due the diapause period. Diapause is an alternative development pathway characterized by profound physiological changes. It is an adaptation from many arthropods to survive during environmental adversities such as cold, lack of resources and the dry season. Diapause is observed in all bee families and occurs mainly during developmental and adult phases. An interesting aspect about diapause in bees is that it may have been a key feature in the evolution of social behavior in this group. One of the hypotheses to explain the evolution of sociality suggests bivoltinism and diapause in adult phase as important factors to the evolution of this phenotype. Therefore, in Chapter 1 we present a review of the phase of life in which diapause occurs among bees and correlated it with variables such as: nest type, voltinism, latitude and social behavior. We found that the life phase in which diapause happens is significant associated with voltinism, latitude and social behavior and that all social bee species have lost diapause during development. To better understand the molecular mechanisms of diapause in T. diversipes, we present in Chapter 2 the genome of this species. We reanalized transcriptomic data previously generated for this species in diapause and identified genomic signatures related to this phenotype. Specifically, the enrichment of transcription factor binding sites in the promotes regions of the differentially expressed genes during diapause. We also compared $T$. diversipes genome with other bee species genome and identifies gain and loss of gene families related with life stage in which diapause occurs. Lastly, we present a byproduct of the $T$. diversipes genome data, the Wolbachia endosymbiont of $T$. diversipes genome and further we performed a population analysis aiming at verifying the presence of this bacteria across five host populations. We identified that all individuals, from all populations, were infected by the same two Wolbachia strains concomitantly. Therefore, this endosymbiont has no effect on the population structure previously described for T. diversipes, neither on sex-biased for male or females described for some of the populations.
\end{abstract}




\section{INTRODUÇÃO GERAL}

\subsection{Diapausa}

Condições ambientais adversas como restrição alimentar, stress e mudanças no fotoperíodo ou temperatura podem culminar em longas mudanças no metabolismo, comportamento, crescimento e desenvolvimento. Essas mudanças, ou estratégias alternativas durante a vida, são conhecidas como hibernação ou diapausa (Fielenbach \& Antebi 2008). A diapausa é caracterizada por mudanças fisiológicas profundas que antecedem às adversidades. Diferentemente da quiescência, a diapausa não é apenas uma diminuição no metabolismo, mas uma via alternativa de desenvolvimento que demanda um metabolismo próprio (Kostál 2006; Hahn \& Denlinger 2011).

A diapausa é amplamente presente entre os artrópodes, e está associada à sobrevivência dos indivíduos durante as estações frias e secas. Esse processo tem sido considerado como um fator importante para o sucesso evolutivo da Classe Insecta (Denlinger 2002). Nos insetos, a diapausa pode ocorrer nas diversas fases do ciclo de vida: embrião, larva, pupa, adulto farato e adulto (Denlinger 2002). Nos estágios iniciais do ciclo de vida ela é denominada de "diapausa do desenvolvimento" e no adulto de "diapausa reprodutiva" (Wolschin \& Gadau 2009). Nos estágios imaturos há uma profunda supressão metabólica. Nos adultos, entretanto, eles podem se mover e se alimentar, apresentando mudanças metabólicas menos profundas (Kostál 2006).

Kostál (2006) divide a diapausa em três fases eco-fisiológicas: pré-diapausa, diapausa e pósdiapausa. Cada fase é regulada pela interação entre fatores endógenos e exógenos (Denlinger 2002). A pré-diapausa é o estágio que antecede a diapausa, nela os indivíduos ainda se mantêm em desenvolvimento ou ativos, mas em resposta a um estímulo externo, o metabolismo é alterado. O estímulo que induz a diapausa é percebido durante um período específico, o qual é determinado geneticamente (Kostál 2006). Dentre os estímulos naturais que induzem a diapausa, o fotoperíodo é o melhor compreendido. Ele é decisivo e comum nas zonas temperadas, mas parece exercer pouca influência nas regiões tropicais (Denlinger 1986; Kostál 2006). Nessas últimas, as mudanças na qualidade do alimento ou fatores de efeito materno podem servir como um estímulo para a indução da diapausa (Denlinger 1986). Na pré-diapausa, pode ocorrer o sequestro de reservas adicionais de gordura, deposição de camadas extras de hidrocarbonetos para impermeabilização da cutícula, ou a síntese de proteínas que permanecem presentes na hemolinfa durante a diapausa (Denlinger 2002). Essa fase é de intensa alimentação e acúmulo de reservas (Kostál 2006). 
A fase de diapausa se inicia quando o desenvolvimento direto ou as atividades cessam, seguido pela supressão metabólica. Mas, em larvas de vida livre e em adultos, que podem se mover, a supressão metabólica é menos profunda e os indivíduos podem continuar ingerindo alimento e estocando energia (Kostál 2006). O início da diapausa pode ocorrer em diferentes períodos do ano, de acordo com cada espécie, e não necessariamente em todos os indivíduos de uma mesma população.

A diapausa termina quando mudanças no ambiente estimulam o restabelecimento do metabolismo normal. Se as condições ambientais forem favoráveis, o desenvolvimento direto ou as atividades são restabelecidas, se não, os indivíduos permanecem em um estado de quiescência, o que caracteriza a pós-diapausa (Kostál 2006). Os insetos nessa fase parecem ser fisiologicamente idênticos aos indivíduos em diapausa, não apresentando mudanças morfológicas perceptíveis, no entanto, a expressão gênica é distinta (Denlinger 2002; Poelchau et al. 2013b; Yocum et al. 2015).

\subsection{Mecanismos moleculares da diapausa em insetos}

Nos últimos anos, com a facilidade de se estudar organismos não-modelo devido aos avanços na tecnologia de sequenciamento de nova geração, muitos estudos têm contribuído para o entendimento dos mecanismos moleculares envolvidos na diapausa (Reynolds et al. 2012; Poelchau et al. 2013a; Amsalem et al. 2015; Santos et al. 2018; Zhai et al. 2019; Zhang et al. 2019). Uma das hipóteses vigentes sugere que as vias metabólicas que regulam a diapausa são conservadas mesmo em espécies filogeneticamente distantes. Sendo assim, um grupo de genes específicos teria um padrão de expressão similar e funções conservadas (Poelchau et al. 2013c). Porém, a existência desse toolkit genético responsável pela diapausa nas diferentes fases de vida e diferentes espécies ainda é controverso (Ragland et al. 2010; Poelchau et al. 2013c; Ragland \& Keep 2017; Santos et al. 2018).

As proteínas heat shock (HSPs) foram as primeiras a serem identificadas como relacionadas aos diferentes tipos de diapausa, desde a dormência de bactérias e fungos à hibernação de mamíferos. Esses resultados fomentaram a hipótese do toolkit genético (Denlinger 2002). Essas proteínas são responsáveis pela manutenção estrutural e estoque de outras proteínas, e atuam na tolerância ao estresse durante a diapausa (revisado por King \& MacRae 2015). Devido às HSPs terem sido identificadas nos diferentes tipos de diapausa, muitos estudos de expressão gênica focaram inicialmente nessas proteínas (Rinehart \& Denlinger 2000; Yocum 2001; Denlinger 2002; 
Yocum et al. 2005, 2006; Tachibana et al. 2005; Robich et al. 2007; Zhang \& Denlinger 2009; Wolschin \& Gadau 2009; Aruda et al. 2011; Gong et al. 2013). No entanto, não foi verificado um padrão conservado na expressão dos genes que as codificam. Por exemplo, sabe-se que a expressão do gene da proteína heat shock HSP90 diminui durante a diapausa na fase de pupa de Sarcophaga crassipalpis (Diptera) (Rinehart \& Denlinger 2000) e de Helicoverpa zea (Lepidoptera) (Zhang \& Denlinger 2009) e na fase larval da vespa Nasonia vitripennis (Wolschin \& Gadau 2009), no entanto, há um aumento na sua expressão durante a diapausa (fase de larva) da abelha solitária Megachile rotundata (Yocum et al. 2005) e de Delia antiqua (Diptera) (fase de pupa) (Chen et al. 2005). O gene $H s p 70$ apresenta aumento em sua expressão durante a diapausa em $S$. crassipalpis (Rinehart et al. 2000), em M. rotundata (Yocum et al. 2005) e na diapausa reprodutiva de Leptinotarsa decemlineata (Coleoptera) (Yocum 2001), mas não há alteração da sua expressão na diapausa na fase adulta de Culex pipiens (Diptera) (Rinehart et al. 2006) e de Drosophila triauria (Goto et al. 1998), na larva de Lucilia sericata (Diptera) (Tachibana et al. 2005) e na pupa de $H$. zea (Zhang \& Denlinger 2009). O gene Hsp23 apresenta sua expressão aumentada em $S$. crassipalpis (Yocum et al. 1998) e em Calliphora vicina (Diptera) (Fremdt et al. 2014), mas não há alteração em L. sericata (Tachibana et al. 2005).

Além das proteínas heat shock, a via de sinalização da insulina, conhecida por regular o metabolismo de carboidrato e gordura em mamíferos, é altamente conservada e uma candidata promissora como reguladora das reservas energéticas durante a diapausa dos insetos (Hahn and Denlinger 2011). Mutações nessa via estão envolvidas com a formação da larva dauer (estágio em diapausa) de Caenorhabditis elegans (Rhabditida) (Kimura et al. 1997) e no controle da diapausa na fase adulta de $C$. pipiens (Sim and Denlinger 2008). Outros dois genes sugeridos como parte do toolkit genético da diapausa são: antígeno nuclear de proliferação celular (pcna) e fosfoenolpiruvato carboxiquinase (pepck). O primeiro é importante para a progressão do ciclo celular e o segundo para o aumento da produção de glicose (Poelchau et al. 2013c). No entanto, estes genes não foram relacionados com a diapausa nas abelhas Megachile rotundata e Tetrapedia diversipes (Yocum et al. 2015; Santos et al. 2018).

Vários outros genes foram identificados como diferencialmente expressos durante a diapausa, e em diversos grupos animais. Esses genes estão principalmente relacionados com: metabolismo energético (Ragland et al. 2010; Bao \& Xu 2011; Pavlides et al. 2011), metabolismo de lipídeos (Ragland et al. 2010; Bao \& Xu 2011; Reynolds et al. 2012; Poelchau et al. 2013b; 2013c), resposta 
ao estresse (Robich et al. 2007; Bao \& Xu 2011; Poelchau et al. 2011, 2013a; Zhang et al. 2013), vias de sinalização (Bao \& Xu 2011; Yocum et al. 2015), citoesqueleto (Kim et al. 2006; Robich et al. 2007; Emerson et al. 2010; Bryon et al. 2013), ciclo celular (Bao \& Xu 2011; Yocum et al. 2015), formação de cutícula (Emerson et al. 2010; Zhang et al. 2013; Poelchau et al. 2013c), metabolismo de hormônios (Bao \& Xu 2011; Poelchau et al. 2011, 2013b) e elementos transponíveis (Robich et al. 2007; Pavlides et al. 2011).

\subsection{A diapausa em Abelhas}

As abelhas constituem um grupo extremamente diverso em número de espécies (aproximadamente 20.000) (Ascher \& Pickering 2018), e de extrema relevância na polinização da vegetação natural e de cultivos. A sua importância econômica também se estende a produtos consumíveis pelos humanos como mel, cera e própolis. Ecológica e evolutivamente falando, são organismos fascinantes, pois apresentam inúmeras adaptações às diversas estruturas florais, habilidade para encontrar alimento e diferentes tipos de matéria prima para construção dos ninhos e habilidade cognitiva para aprender e memorizar onde esses recursos foram encontrados (Michener 2007). A característica mais intrigante desses insetos é a diversidade na sua organização social, variando desde espécies solitárias a altamente eussociais (Michener 2007). Embora a maioria das espécies de abelhas seja solitária, ainda assim é o grupo que apresenta a maior diversidade de tipos de organização social entre os animais (Richards 2019).

Abelhas solitárias constroem o próprio ninho e alimentam as crias sem ajuda de outras abelhas, geralmente morrem antes do desenvolvimento e emergência da prole. As abelhas sociais podem ser classificadas em: sub-sociais - a fêmea adulta desempenha cuidado materno prolongado; primitivamente eussociais - possuem duas castas, rainha e operária, que diferem principalmente na fisiologia e comportamento, formam colônias menores e temporárias que são fundadas por uma fêmea solitária; e altamente eussociais - possuem duas castas morfologicamente distintas, reprodutiva e operária, que não vivem independente uma da outra. Formam colônias grandes e perenes (Michener 2007; Rehan \& Toth 2015).

$\mathrm{O}$ fenômeno da diapausa tem sido reportado para as abelhas independentemente da organização social. A diapausa ocorre principalmente nos estágios de pré-pupa e adulto, e ao contrário de outros insetos, uma mesma espécie pode ter indivíduos em diapausa em fases diferentes do ciclo de vida, como resposta ao mesmo estímulo do ambiente (revisado por Santos 
et al. 2019). A diapausa na fase adulta apresenta fenótipos variáveis. Há espécies que permanecem dormentes em um casulo antes da emergência (Wasielewski et al. 2013), outras que copulam e permanecem em cavidades no solo ou na madeira durante o inverno (Amsalem et al. 2015; Rehan et al. 2016) e espécies que apenas diminuem as atividades, sendo a dormência não tão profunda (Dos-Santos et al. 2014). Chamaremos aqui de "diapausa na fase adulta" aquela em que ocorrem mudanças metabólicas mais profundas e de "diapausa reprodutiva" aquela em que as atividades apenas diminuem (Santos et al. 2019).

Outro aspecto interessante sobre diapausa em abelhas é que ela pode ter sido fundamental para a evolução do comportamento social. Há várias hipóteses na literatura acerca desse tópico, uma delas é a diapause ground plan. Essa hipótese foi inicialmente proposta para vespas de clima temperado, sugerindo que as vias metabólicas da diapausa e não diapausa teriam sido cooptadas para os fenótipos de operária e rainha (Hunt 2006). Ainda, sugere que o bivoltismo e a diapausa na fase adulta seriam pré-requisitos para evolução da socialidade (Hunt \& Amdam 2005).

Essa hipótese tem sido muito debatida, e vem ganhando mais suporte à medida que novas evidências vêm sendo descritas, como por exemplo nos estudos com Polistes metricus (Hunt et al. 2007) e Bombus terrestris (Amsalem et al. 2015). Em Polistes, o tempo prolongado de desenvolvimento e o sequestro de hexamerina (proteína de armazenamento) (duas assinaturas de diapausa) caracterizaram o desenvolvimento das rainhas virgens. Em Bombus foi descrita uma sobreposição de mecanismos genéticos que controlam a diapausa e a determinação de castas (Amsalem et al. 2015), e foi demonstrado que as operárias que emergem tardiamente apresentam assinaturas de diapausa em comparação com as operárias que emergem mais cedo. Entre os genes diferencialmente expressos entre operárias precoces e tardias estavam hexamerinal, pepck e mfe (Treanore et al. 2019).

Em uma revisão recente (Santos et al., 2019, Capítulo 1 desta tese), mostramos que a fase em que a diapausa ocorre está significativamente correlacionada ao nível de socialidade. Em todas as linhagens em que o comportamento social evoluiu, a diapausa na fase do desenvolvimento foi perdida. Mais especificamente temos que a diapausa na fase adulta, antes do acasalamento, ocorre em espécies sub-sociais, após o acasalamento em espécies primitivamente eussociais e a diapausa reprodutiva em espécies altamente eussociais (Santos et al. 2019). Essas evidências corroboram a hipótese do ground plan diapause e sugere que os mecanismos moleculares que regulam as 
mudanças evolutivas na fase em que a diapausa ocorre podem ter sido cooptados durante a evolução da socialidade.

\subsection{A espécie modelo Tetrapedia diversipes}

Tetrapedia diversipes é uma espécie de abelha solitária, coletora de óleo e presente na região Neotropical. Nos últimos anos tem sido nossa espécie modelo para a compreensão dos mecanismos moleculares envolvidos na diapausa do desenvolvimento. O gênero Tetrapedia faz parte da tribo Tetrapediini, a qual foi recentemente incluída na sub-família Xylocopinae (Bossert et al. 2018).

T. diversipes nidifica em cavidades estreitas pré-existentes na natureza, como por exemplo orifícios em troncos previamente utilizados por besouros, e também em ninhos-armadilha (Alvesdos-Santos et al. 2002). O índice de nidificação em ninhos-armadilha é alto, o que faz dessa espécie uma excelente candidata, ou modelo, para estudos envolvendo genética, ecologia e evolução (Menezes et al. 2012; Neves et al. 2012, 2014; Pinto et al. 2015; Rocha-Filho \& Garófalo 2015; Arias et al. 2016).

Os ninhos são construídos em forma de tubo. Após a célula ser aprovisionada com uma massa de pólen misturada a óleo, o ovo é colocado sobre esse alimento. Uma camada de grãos finos de areia misturados a óleo floral é utilizada para o fechamento dessa célula, e que serve de base para a célula seguinte. Cada ninho pode ter um número diferente de células, variando de uma a sete. É comum a reutilização dos ninhos antigos pelas gerações subsequentes, ou a ocupação de um mesmo ninho por outra fêmea, no caso do mesmo ter sido abandonado antes de ser finalizado (quando a fêmea morre durante o forrageamento, por exemplo) (Alves-dos-Santos et al. 2002).

A espécie $T$. diversipes apresenta duas gerações anuais, ou seja, é bivoltina. Na primeira geração (ninhos iniciados na primavera e verão) os indivíduos têm o desenvolvimento contínuo e, portanto, é mais rápido em relação à segunda geração (ninhos iniciados no final do verão e no outono). Nesta, os indivíduos entram em diapausa no estágio de larva madura (estágio larval que precede a formação da pupa), também chamado de $5^{\circ}$ instar larval, e o tempo total de desenvolvimento é mais longo (Alves-dos-santos et al. 2007). Essa parada do desenvolvimento ocorre nos meses mais frios e secos (junho e julho) e pode variar de 21 a 366 dias, o que indica que o período de diapausa pode ser longo (Cordeiro 2009). Portanto, essa espécie é um modelo interessante para se estudar esse fenômeno, pois apresenta os dois fenótipos (diapausa e não diapausa) bem demarcados a depender da época do ano em que o desenvolvimento larval ocorre. 
Em estudo prévio, com o objetivo de entender molecularmente a diapausa em $T$. diversipes, foram obtidos dados do perfil da expressão gênica de larvas em diapausa e não-diapausa (Santos et al. 2018). Foram identificados 2.274 genes diferencialmente expressos. Esses genes estão associados à desintoxicação celular, formação de cutícula, rearranjos do citoesqueleto, metabolismo de lipídeos e esteróis e ao ciclo celular. Vários desses genes já foram discutidos previamente como sendo importantes para a diapausa (Liu et al. 2005; Carrasco et al. 2011; Podrabsky \& Culpepper 2012; Bryon et al. 2013), não somente em insetos, mas também em Caenorhabditis elegans quanto à formação da larva dauer (estágio em diapausa) (McElwee et al. 2004; Li et al. 2004).

\section{Justificativa}

Os rápidos avanços na tecnologia de sequenciamento de nova geração estão ampliando as oportunidades de esclarecer as bases moleculares de fenótipos complexos, e ampliando as possibilidades de realizar estudos em organismos não-modelo (Poelchau et al. 2013b). O sequenciamento de genomas, em contraste a dados de sequências de loci individuais, revela como o material genético está organizado, os tipos e abundância de elementos móveis, RNAs não codificantes de função regulatória e marcadores de cromatina, genes específicos que são fundamentais a nível fenotípico, a aquisição de novos genes e a modificação dos existentes por seleção positiva demonstrando adaptação ao nível genômico. Esses dados podem também contribuir, de forma geral, para estudos evolutivos em grande escala (Ellegren 2014). Além disso, a disponibilidade de um genoma permite estudos que relacionem metilação, splicing alternativo e fenótipos, como já realizado em A. mellifera, espécie que tem se tornado modelo para estudos de metilação do DNA em insetos (Glastad et al. 2011).

Atualmente, estão disponibilizados no banco de dados "National Center for Biotechnology Information" (NCBI), 97 genomas de Hymenoptera, sendo 18 de abelhas: Apis mellifera (Elsik et al. 2014), Apis cerana (Park et al. 2015), Apis dorsata (Oppenheim et al. 2019), Apis florea (Woodard et al. 2011), Bombus impatiens, Bombus terrestris (Sadd et al. 2015), Ceratina australiensis (Rehan et al. 2018), Ceratina calcarata (Rehan et al. 2016), Dufourea novaeangliae (Kapheim et al. 2015), Euglossa dilemma (Brand et al. 2017), Eufriesea mexicana, Habropoda laboriosa (Kapheim et al. 2015), Lasioglossum albipes (Kocher et al. 2013), Lepidotrigona ventralis, Megachile rotundata (Woodard et al. 2011), Melipona quadrifasciata, Nomia melanderi 
(Kapheim et al. 2019) e Osmia bicornis (Beadle et al. 2019) (NCBI, http://www.ncbi.nlm.nih.gov/).

A comparação de genomas tem resultado em avanços significativos na compreensão de fenótipos complexos. Na área da diapausa, a comparação do genoma de L. albipes com A. mellifera identificou expansão em uma família gênica que pode estar relacionada à diapausa obrigatória das fundadoras de L .albipes (Kocher et al. 2013). Na sociobiologia, um estudo comparativo entre os genomas de 10 abelhas, que apresentam diferentes níveis de socialidade, revelou que as transições entre níveis de socialidade são acompanhadas de uma maior complexidade na regulação gênica (ganho de sítios de ligação para fatores de transcrição na região promotora de genes) e diminuição na abundância de elementos transponíveis (Kapheim et al. 2015). Embora esse banco de dados atual já permita algumas análises comparativas, há um viés negativo quanto à representatividade das abelhas solitárias. Dos 18 genomas publicados somente seis são de abelhas solitárias. Aumentar a representatividade de genomas das espécies solitárias, pois constituem a maioria das espécies, irá contribuir para a compreensão de fenótipos complexos. Além da caracterização e comparação entre genomas, o sequenciamento de nova geração permite a análise de reads “contaminantes" que podem nos dar informações sobre as interações inseto-planta (Araujo et al. 2016), assim como de parasitas e simbiontes (Dhaygude et al. 2019).

\section{Referências Bibliográficas}

Alves-dos-santos I, Machado IC, Gaglianone MC (2007) História natural das abelhas coletoras de óleo. Oecologia Bras 11:544-557

Alves-dos-Santos I, Melo GAR, Rozen JR JG (2002) Biology and immature stages of the bee tribe Tetrapediini (Hymenoptera: Apidae). Am Museum Novit 3377:1-45

Amsalem E, Galbraith DA, Cnaani J, et al (2015) Conservation and modification of genetic and physiological toolkits underpinning diapause in bumble bee queens. Mol Ecol 24:5596-5615. doi: $10.1111 / \mathrm{mec} .13410$

Araujo NS, Zuntini AR, Arias MC (2016) Getting usefull information from RNA-Seq contaminants: A case of study in the oil-collecting bee Tetrapedia diversipes transcriptome. Omi A J Integr Biol 20:1-2. doi: 10.1089/omi.2016.0054

Arias MC, Aulagnier S, Baerwald EF, et al (2016) Microsatellite records for volume 8, issue 1. Conserv Genet Resour 8:43-81. doi: 10.1007/s12686-016-0522-2

Aruda AM, Baumgartner MF, Reitzel AM, Tarrant AM (2011) Heat shock protein expression during stress and diapause in the marine copepod Calanus finmarchicus. J Insect Physiol 57:665-675. doi: 10.1016/j.jinsphys.2011.03.007

Ascher JS, Pickering J (2018) Discover Life bee species guide and world checklist (Hymenoptera: Apoidea). Available

at: <https://www.discoverlife.org/mp/20q?guide=Apoidea_species\&flags=HAS:> 
Bao B, Xu W-H (2011) Identification of gene expression changes associated with the initiation of diapause in the brain of the cotton bollworm, Helicoverpa armigera. BMC Genomics 12:114. doi: $10.1186 / 1471-2164-12-224$

Beadle K, Singh KS, Troczka BJ, et al (2019) Genomic insights into neonicotinoid sensitivity in the solitary bee Osmia bicornis. PLoS Genet 15:1-19. doi: 10.1371/journal.pgen.1007903

Bossert S, Murray EA, Almeida EAB, et al (2018) Combining transcriptomes and ultraconserved elements to illuminate the phylogeny of Apidae. Mol Phylogenet Evol 130:121-131. doi: 10.1016/j.ympev.2018.10.012

Brand P, Saleh N, Pan H, et al (2017) The Nuclear and Mitochondrial Genomes of the Facultatively Eusocial Orchid Bee Euglossa dilemma. G3 Genes, Genomes, Genet 7:1-18. doi: 10.1534/g3.117.043687

Bryon A, Wybouw N, Dermauw W, et al (2013) Genome wide gene-expression analysis of facultative reproductive diapause in the two-spotted spider mite Tetranychus urticae. BMC Genomics 14:815-835. doi: 10.1186/1471-2164-14-815

Carrasco MA, Buechler SA, Arnold RJ, et al (2011) Elucidating the biochemical overwintering adaptations of larval Cucujus clavipes puniceus, a nonmodel organism, via high throughput proteomics. J Proteome Res 10:4634-4646

Chen B, Kayukawa T, Monteiro A, Ishikawa Y (2005) The expression of the HSP90 gene in response to winter and summer diapauses and thermal-stress in the onion maggot, Delia antiqua. Insect Mol Biol 14:697-702. doi: 10.1111/j.1365-2583.2005.00602.x

Cordeiro GD (2009) Abelhas solitárias nidificantes em ninhos-armadilha em quatro áreas de Mata Atlântica do Estado de São Paulo. Universidade de São Paulo

Denlinger DL (2002) Regulation of Diapause. Annu Rev Entomol 47:93-122

Denlinger DL (1986) Dormancy in tropical insects. Annu Rev Entomol 31:239-264. doi: 10.1146/annurev.en.31.010186.001323

Dhaygude K, Nair A, Johansson H, et al (2019) The first draft genomes of the ant Formica exsecta, and its Wolbachia endosymbiont reveal extensive gene transfer from endosymbiont to host. BMC Genomics 20:1-16. doi: 10.1186/s12864-019-5665-6

Dos-Santos CF, Nunes-Silva P, Halinski R, Blochtein B (2014) Diapause in Stingless Bees (Hymenoptera: Apidae). Sociobiology 61:369-377. doi: 10.13102/sociobiology.v61i4.369377

Ellegren H (2014) Genome sequencing and population genomics in non-model organisms. Trends Ecol Evol 29:51-63. doi: 10.1016/j.tree.2013.09.008

Elsik CG, Worley KC, Bennett AK, et al (2014) Finding the missing honey bee genes : lessons learned from a genome upgrade. BMC Genomics 15:86-115. doi: 10.1186/1471-2164-15-86

Emerson KJ, Bradshaw WE, Holzapfel CM (2010) Microarrays reveal early transcriptional events during the termination of larval diapause in natural populations of the mosquito, Wyeomyia smithii. PLoS One 5:1-10. doi: 10.1371/journal.pone.0009574

Fielenbach N, Antebi A (2008) C. elegans dauer formation and the molecular basis of plasticity. Genes Dev 22:2149-2165. doi: 10.1101/gad.1701508

Fremdt H, Amendt J, Zehner R (2014) Diapause-specific gene expression in Calliphora vicina (Diptera: Calliphoridae) -- a useful diagnostic tool for forensic entomology. Int J Legal Med 128:1001-1011. doi: 10.1007/s00414-013-0920-x

Glastad KM, Hunt BG, Yi SV, Goodisman MA. (2011) DNA methylation in insects: on the brink of the epigenomic era. Insect Mol Biol 20:553-565. doi: 10.1111/j.1365-2583.2011.01092.x

Gong Z-J, Wu Y-Q, Miao J, et al (2013) Global transcriptome analysis of orange wheat blossom 
midge, Sitodiplosis mosellana (Gehin) (Diptera: Cecidomyiidae) to identify candidate transcripts regulating diapause. PLoS One 8:1-11. doi: 10.1371/journal.pone.0071564

Goto SG, Yoshida KM, Kimura MT (1998) Accumulation of Hsp70 mRNA under environmental stresses in diapausing and nondiapausing adults of Drosophila triauraria. J Insect Physiol 44:1009-1015. doi: 10.1016/S0022-1910(97)00143-1

Hahn DA, Denlinger DL (2011) Energetics of Insect Diapause. Annu Rev Entomol 56:103-121. doi: 10.1146/annurev-ento-112408-085436

Hunt JH (2006) Evolution of castes in Polistes. Ann Zool Fennici 43:407-422. doi: $10.2307 / 23736751$

Hunt JH, Amdam GV (2005) Bivoltinism as an antecedent to eusociality in the paper wasp genus Polistes. Science 308:264-267. doi: 10.1126/science.1109724

Hunt JH, Kensinger BJ, Kossuth JA, et al (2007) A diapause pathway underlies the gyne phenotype in Polistes wasps, revealing an evolutionary route to caste-containing insect societies. Proc Natl Acad Sci U S A 104:14020-14025. doi: 10.1073/pnas.0705660104

Kapheim KM, Pan H, Li C, et al (2015) Genomic signatures of evolutionary transitions from solitary to group living. Science 348:1139-1144

Kapheim KM, Pan H, Li C, et al (2019) Draft genome assembly and population genetics of an agricultural pollinator, the solitary alkali bee (Halictidae: Nomia melanderi). G3 Genes, Genomes, Genet 9:625-634. doi: 10.1534/g3.118.200865

Kim M, Robich RM, Rinehart JP, Denlinger DL (2006) Upregulation of two actin genes and redistribution of actin during diapause and cold stress in the northen house mosquito, Culex pipiens. J Insect Physiol 52:1226-1233

Kimura KD, Tissenbaum HA, Liu Y, Ruvkun G (1997) daf-2 , an Insulin Receptor - Like Gene That Regulates Longevity and Diapause in Caenorhabditis elegans. Science 277:942-946. doi: 10.1126/science.277.5328.942

King AM, MacRae TH (2015) Insect Heat Shock Proteins during stress and diapause. Annu Rev Entomol 60:59-75. doi: 10.1146/annurev-ento-011613-162107

Kocher SD, Li C, Yang W, et al (2013) The draft genome of a socially polymorphic halictid bee, Lasioglossum albipes. Genome Biol 14:1-14. doi: 10.1186/gb-2013-14-12-r142

Kostál V (2006) Eco-physiological phases of insect diapause. J Insect Physiol 52:113-127. doi: 10.1016/j.jinsphys.2005.09.008

Li J, Brown G, Ailion M, et al (2004) NCR-1 and NCR-2, the C. elegans homologs of the human Niemann-Pick type C1 disease protein, function upstream of DAF-9 in the dauer formation pathways. Development 131:5741-5752. doi: 10.1242/dev.01408

Liu M, Zhang T-Y, Xu W-H (2005) A cDNA encoding diazepam-binding inhibitor/acyl-CoAbinding protein in Helicoverpa armigera: molecular characterization and expression analysis associated with pupal diapause. Comp Biochem Physiol Part C 141:168-176. doi: 10.1016/j.cca.2005.05.011

McElwee JJ, Schuster E, Blanc E, et al (2004) Shared transcriptional signature in Caenorhabditis elegans dauer larvae and long-lived daf-2 mutants implicates detoxification system in longevity assurance. J Biol Chem 279:44533-44543. doi: 10.1074/jbc.M406207200

Menezes GB, Gonçalves-Esteves V, Bastos EMAF, et al (2012) Nesting and use of pollen resources by Tetrapedia diversipes Klug (Apidae) in Atlantic Forest areas (Rio de Janeiro, Brazil) in different stages of regeneration. Rev Bras Entomol 56:86-94

Michener CD (2007) The Bees of the World, 2nd edn. The Johns Hopkins University Press, Baltimore 
Neves CML, Carvalho CAL, Machado CS, et al (2014) Pollen consumed by the solitary bee Tetrapedia diversipes (Apidae:Tetrapediini) in a tropical agroecosystem. Grana 53:302-308. doi: 10.1080/00173134.2014.931455

Neves CML, Carvalho CAL, Souza AV, Lima CA (2012) Morphometric characterization of a population of Tetrapedia diversipes in restricted areas in Bahia, Brazil (Hymenoptera: Apidae). Sociobiology 59:767-782

Oppenheim SJ, Cao X, Rueppel O, et al (2019) Whole Genome Sequencing and Assembly of the Asian Honey Bee Apis dorsata. Genome Biol Evol 1-22

Park D, Jung WW, Choi BS, et al (2015) Uncovering the novel characteristics of Asian honey bee, Apis cerana, by whole genome sequencing. BMC Genomics 16:1-16. doi: 10.1186/14712164-16-1

Pavlides SC, Pavlides SA, Tammariello SP (2011) Proteomic and phosphoproteomic profiling during diapause entrance in the flesh fly, Sarcophaga crassipalpis. J Insect Physiol 57:635644. doi: 10.1016/j.jinsphys.2011.03.022

Pinto CE, da Silva A, Cordeiro GD, Alves-dos-Santos I (2015) The body size of the oil-collecting bee Tetrapedia diversipes (Apidae). J Hymenopt Res 47:103-113. doi: 10.3897/JHR.47.4837

Podrabsky JE, Culpepper KM (2012) Cell cycle regulation during development and dormancy in embryos of the annual killifish Austrofundulus limnaeus. Cell Cycle 11:1697-1704. doi: $10.4161 /$ cc. 19881

Poelchau MF, Reynolds JA, Denlinger DL, et al (2013a) Transcriptome sequencing as a platform to elucidate molecular components of the diapause response in the Asian tiger mosquito, Aedes albopictus. Physiol Entomol 38:173-181. doi: 10.1111/phen.12016

Poelchau MF, Reynolds JA, Denlinger DL, et al (2011) A de novo transcriptome of the Asian tiger mosquito, Aedes albopictus, to identify candidate transcripts for diapause preparation. BMC Genomics 12:1-19. doi: 10.1186/1471-2164-12-619

Poelchau MF, Reynolds JA, Elsik CG, et al (2013b) RNA-Seq reveals early distinctions and late convergence of gene expression between diapause and quiescence in the Asian tiger mosquito, Aedes albopictus. J Exp Biol 216:4082-4090. doi: 10.1242/jeb.089508

Poelchau MF, Reynolds JA, Elsik CG, et al (2013c) Deep sequencing reveals complex mechanisms of diapause preparation in the invasive mosquito, Aedes albopictus. Proc R Soc B Biol Sci 280:1-9. doi: 10.1098/rspb.2013.0143

Ragland GJ, Denlinger DL, Hahn DA (2010) Mechanisms of suspended animation are revealed by transcript profiling of diapause in the flesh fly. Proc Natl Acad Sci U S A 107:1490914914. doi: 10.1073/pnas.1007075107

Ragland GJ, Keep E (2017) Comparative transcriptomics support evolutionary convergence of diapause responses across Insecta. Physiol Entomol 42:246-256. doi: 10.1111/phen.12193

Rehan SM, Glastad KM, Lawson SP, Hunt BG (2016) The genome and methylome of a subsocial small carpenter bee, Ceratina calcarata. Genome Biol Evol 8:1401-1410. doi: 10.1093/gbe/evw079

Rehan SM, Glastad KM, Steffen MA, et al (2018) Conserved genes underlie phenotypic plasticity in an incipiently social bee. Genome Biol Evol 10:2749-2758. doi: 10.1093/gbe/evy212

Rehan SM, Toth AL (2015) Climbing the social ladder: the molecular evolution of sociality. Trends Ecol Evol 30:426-433. doi: 10.1016/j.tree.2015.05.004

Reynolds JA, Poelchau MF, Rahman Z, et al (2012) Transcript profiling reveals mechanisms for lipid conservation during diapause in the mosquito, Aedes albopictus. J Insect Physiol 58:966-973. doi: 10.1016/j.jinsphys.2012.04.013 
Richards MH (2019) Social trait definitions influence evolutionary inferences: a phylogenetic approach to improving social terminology for bees. Curr Opin Insect Sci 34:97-104. doi: 10.1016/j.cois.2019.04.006

Rinehart J, Yocum G, Denlinger D (2000) Developmental upregulation of inducible hsp70 transcripts, but not the cognate form, during pupal diapause in the flesh fly, Sarcophaga crassipalpis. Insect Biochem Mol Biol 30:515-521. doi: 10.1016/S0965-1748(00)00021-7

Rinehart JP, Denlinger DL (2000) Heat-shock protein 90 is down-regulated during pupal diapause in the flesh fly, Sarcophaga crassipalpis, but remains responsive to thermal stress. Insect Mol Biol 9:641-645. doi: 10.1046/j.1365-2583.2000.00230.x

Rinehart JP, Robich RM, Denlinger DL (2006) Enhanced Cold and Desiccation Tolerance in Diapausing Adults of Culex pipiens, and a Role for Hsp70 in Response to Cold Shock but Not as a Component of the Diapause Program. J Med Entomol 43:713-722. doi: 10.1093/jmedent/43.4.713

Robich RM, Rinehart JP, Kitchen LJ, Denlinger DL (2007) Diapause-specific gene expression in the northern house mosquito, Culex pipiens L., identified by supressive subtractive hybridization. J Insect Physiol 53:235-245

Rocha-Filho LC, Garófalo CA (2015) Natural history of Tetrapedia diversipes (Hymenoptera: Apidae) in an Atlantic Semideciduous Forest remnant surrounded by coffee crops, Coffea arabica (Rubiaceae). Ann Entomol Soc Am 0:1-15. doi: 10.1093/aesa/sav153

Sadd BM, Barribeau SM, Bloch G, et al (2015) The genomes of two key bumblebee species with primitive eusocial organization. Genome Biol 16:16-76. doi: 10.1186/s13059-015-0623-3

Santos PKF, Araujo NS, Françoso E, et al (2018) Diapause in a tropical oil-collecting bee: Molecular basis unveiled by RNA-Seq. BMC Genomics 19:1-11. doi: 10.1186/s12864-0184694-X

Santos PKF, Arias MC, Kapheim KM (2019) Loss of developmental diapause as a prerequisite for social evolution in bees. Biol Lett 15:1-7. doi: 10.1101/649897

Sim C, Denlinger DL (2008) Insulin signaling and FOXO regulate the overwintering diapause of the mosquito Culex pipiens. Proc Natl Acad Sci U S A 105:6777-6781. doi: 10.1073/pnas.0802067105

Tachibana S-I, Numata H, Goto SG (2005) Gene expression of heat-shock proteins (Hsp23, Hsp70 and $H$ sp90) during and after larval diapause in the blow fly Lucilia sericata. J Insect Physiol 51:641-647. doi: 10.1016/j.jinsphys.2004.11.012

Treanore ED, Kiner JM, Kerner ME, Amsalem E (2019) The role of insect diapause in the divergence of social female castes: Insights from primitively eusocial bumble bee workers. bioRxiv. doi: http://dx.doi.org/10.1101/758367

Wasielewski O, Wojciechowicz T, Giejdasz K, Krishnan N (2013) Overwintering strategies in the red mason solitary bee - Physiological correlates of midgut metabolic activity and turnover of nutrient reserves in females of Osmia bicornis. Apidologie 44:642-656. doi: $10.1007 / \mathrm{s} 13592-013-0213-\mathrm{x}$

Wolschin F, Gadau J (2009) Deciphering proteomic signatures of early diapause in Nasonia. PLoS One 4:1-8. doi: 10.1371/journal.pone.0006394

Woodard SH, Fischman BJ, Venkat A, et al (2011) Genes involved in convergent evolution of eusociality in bees. Proc Natl Acad Sci 108:7472-7477. doi: 10.1073/pnas.1103457108

Yocum GD (2001) Differential expression of two HSP70 transcripts in response to cold shock, thermoperiod, and adult diapause in the Colorado potato beetle. J Insect Physiol 47:11391145. doi: 10.1016/S0022-1910(01)00095-6 
Yocum GD, Joplin KH, Denlinger DL (1998) Upregulation of a $23 \mathrm{kDa}$ small heat shock protein transcript during pupal diapause in the flesh fly, Sarcophaga crassipalpis. Insect Biochem Mol Biol 28:677-682. doi: 10.1016/S0965-1748(98)00046-0

Yocum GD, Kemp WP, Bosch J, Knoblett JN (2005) Temporal variation in overwintering gene expression and respiration in the solitary bee Megachile rotundata. J Insect Physiol 51:621629

Yocum GD, Kemp WP, Bosch J, Knoblett JN (2006) Thermal history influences diapause development in the solitary bee Megachile rotundata. J Insect Physiol 52:1113-1120. doi: 10.1016/j.jinsphys.2006.07.010

Yocum GD, Rinehart JP, Horvath DP, et al (2015) Key molecular processes of the diapause to post-diapause quiescence transition in the alfalfa leafcutting bee Megachile rotundata identified by comparative transcriptome analysis. Physiol Entomol 40:103-112. doi: 10.1111/phen. 12093

Zhai Y, Dong X, Gao H, et al (2019) Quantitative proteomic and transcriptomic analyses of metabolic regulation of adult reproductive diapause in Drosophila suzukii (Diptera: Drosophilidae) females. Front Physiol 10:1-11. doi: 10.3389/FPHYS.2019.00344

Zhang C, Wei D, Shi G, et al (2019) Understanding the regulation of overwintering diapause molecular mechanisms in Culex pipiens pallens through comparative proteomics. Sci Rep 9:1-12. doi: 10.1038/s41598-019-42961-w

Zhang Q, Denlinger DL (2009) Molecular characterization of heat shock protein 90, 70 and 70 cognate cDNAs and their expression patterns during thermal stress and pupal diapause in the corn earworm. J Insect Physiol 56:138-150. doi: 10.1016/j.jinsphys.2009.09.013

Zhang Q, Lu Y-X, Xu W-H (2013) Proteomic and metabolomic profiles of larval hemolymph associated with diapause in the cotton bollworm, Helicoverpa armigera. BMC Genomics 14:1-13. doi: 10.1186/1471-2164-14-751 


\section{HISTÓRICO DA TESE E CONCLUSÕES GERAIS}

A história desta tese começa em 2002, quando um fascinante trabalho sobre a história natural de Tetrapedia diversipes foi publicado pela Dra. Isabel Alves dos Santos e colaboradores (Alves-dos-Santos et al. 2002). Neste trabalho, foi descrito cada detalhe sobre adaptações morfológicas, coleta de alimento e materiais para construção do ninho, tempo de desenvolvimento e semelhanças e diferenças com a espécie cleptoparasita Coelioxoides waltheriae. Mas foi apenas em 2013, início do meu mestrado que li pela primeira vez este trabalho.

No início do meu mestrado, estava empolgada com a possibilidade de participar de um projeto que fosse parte da nova linha de pesquisa que se instaurava no laboratório: o uso de dados de sequenciamento de nova geração, especificamente dados de transcriptoma, para compreensão de fenótipos complexos. Para que isso fosse possível, comecei a estudar características das espécies que já eram foco de outros projetos em desenvolvimento no laboratório. Foi quando o termo diapausa me chamou atenção no trabalho sobre T. diversipes (Alves-dos-Santos et al. 2002). Especificamente por ser um fenótipo que aparecia apenas em alguns indivíduos. Conversei com meus colegas e minha orientadora Dra. Maria Cristina Arias e então surgiu a ideia de tentar entender os mecanismos moleculares da diapausa em $T$. diversipes.

Eu nunca tinha escutado ou lido nada relacionado à diapausa, mas desde o momento em que eu descobri o que era, achei que era um dos fenótipos mais incríveis existentes na natureza. Quando comecei a ler sobre diapausa, vi que era um assunto estudado há décadas, principalmente usando como modelo espécies de clima temperado. Sendo que pouquíssimas espécies de abelhas faziam parte desses estudos. Apesar de avanços no conhecimento sobre os mecanismos moleculares da diapausa, ainda hoje não é consenso na literatura se as vias metabólicas regulando este fenótipo são conservadas entre diferentes espécies.

No meu mestrado então identificamos diversos genes relacionados à diapausa em $T$. diversipes (Santos et al. 2018). Além de gerarmos este conhecimento para uma espécie de abelha Neotropical, coletamos os indivíduos em ambiente natural, sem a indução da diapausa no laboratório. Estudos recentes demonstram que a variação de expressão gênica é diferente quando a diapausa é induzida em laboratório ou coletada em ambientes naturais. Mas gostaríamos de ir além, gostaríamos de compreender melhor como esses genes são regulados, compreender os mecanismos epigenéticos envolvidos na tradução do sinal ambiental em mudanças de expressão 
gênica, identificar genes candidatos e realizar estudos funcionais. Para isso, seria essencial a disponibilidade de um genoma da espécie.

Além das características comportamentais e de história de vida únicas de T. diversipes, o fato desta espécie ser muito fácil de observar e coletar, principalmente por ser uma das mais abundante em ninhos-armadilhas em diferentes áreas da sua distribuição, nos motivou a sequenciar este genoma. $\mathrm{O}$ genoma de $T$. diversipes é o primeiro sequenciado de uma espécie de abelha solitária da região Neotropical. Esperamos que possa ser referência para outras áreas de interesse nos estudos sobre abelhas.

$\mathrm{Na}$ transição entre o mestrado e doutorado, tive conhecimento sobre a diapausa ser importante para uma das hipóteses vigentes que explicam a evolução do comportamento social em Hymenoptera. Especificamente, bivoltismo e diapausa na fase adulta teriam sido mudanças necessárias na transição da vida solitária para vida social em vespas (Hunt \& Amdam 2005). Achei essa hipótese muito interessante, e para mim fazia sentido, pois havia observado que quando as espécies sociais apresentavam diapausa, era sempre na fase adulta. Desde então eu tinha pensando em fazer uma revisão da literatura sobre diapausa em abelhas para ver se esse padrão realmente existia. No entanto, por não ser da área que estuda evolução da socialidade, descobri algum tempo depois que essa hipótese era muito criticada. Especificamente em um evento científico, observei pesquisadores questionando a validade da hipótese para espécies tropicais, pois a maioria delas não apresenta diapausa. Isso me motivou mais ainda a realizar esta revisão. Este período coincidiu com o tempo em que realizei o doutorado sanduíche no laboratório da Dra. Karen Kapheim na Utah State University. Ela deu apoio ao desenvolvimento deste trabalho e sugeriu as análises para dar suporte estatístico aos resultados. Este trabalho resultou no capítulo 1 da tese.

Neste capítulo 1, fizemos uma revisão da fase de vida em que a diapausa ocorre em diferentes espécies de abelhas, e correlacionamos a fase da diapausa com diferentes aspectos de vida. Os nossos resultados mostram que o ancestral do grupo das abelhas provavelmente tinha diapausa na fase do desenvolvimento e que em todas as vezes que a socialidade surgiu nesse grupo, a diapausa foi perdida ou passou a acontecer na fase adulta. Além disso, a fase em que a diapausa ocorre é significativamente relacionada ao voltismo e latitude (Santos et al. 2019). Estes resultados são importantes pois estão de acordo com a diapause ground plan, que sugere que vias metabólicas da diapausa foram co-optadas na evolução da socialidade. Com nossa revisão mostramos que há uma sobreposição entre as fases que a diapausa ocorre e o nível de socialidade que uma espécie 
apresenta. Entender as mudanças moleculares relacionadas com a mudança de fase da diapausa pode contribuir com o entendimento da evolução do comportamento social, que não tem sido estudado sob esta perspectiva.

De volta ao Brasil, focamos no principal objetivo deste trabalho, que era gerar o genoma de $T$. diversipes e identificar assinaturas genômicas relacionadas à diapausa nesta espécie. Mostramos que o genoma de $T$. diversipes é rico em elementos transponíveis, assim como outras espécies de abelhas solitárias. Também apresentou transferência lateral de genes de origem mitocondrial e bacteriana. Na reanálise dos dados de transcriptoma de $T$. diversipes em diapausa e não diapausa, associados à disponibilidade do genoma, pudemos identificar enriquecimento de fatores de transcrição na região promotora dos genes relacionados à diapausa. Também ampliamos as análises para identificar quais genes são centrais dentre os diferencialmente expressos e encontramos que para o grupo de genes envolvidos na formação de cutícula e digestão, o padrão de expressão pode variar no início do desenvolvimento, quando comparamos as duas gerações. Além disso, comparamos o genoma de $T$. diversipes com genomas de outras espécies de abelhas que também apresentam diapausa na fase de desenvolvimento, e outras na fase adulta. Com esta abordagem identificamos ganhos e perdas de famílias gênicas relacionadas às diferentes fases em que a diapausa ocorre. Estes resultados sugerem que há diferenças genéticas relacionadas a essas fases e a diapausa, e que o grupo das abelhas é um bom modelo para estender estas análises e compreender as bases conservadas da diapausa em diferentes espécies.

Durante o meu doutorado, tive oportunidade de fazer uma disciplina chamada "Plasticidade Ontogenética, Epigênese e Evolução". Nela eu ouvi falar sobre Wolbachia, e fiquei fascinada pelos efeitos que uma bactéria poderia causar na reprodução do hospedeiro. Lembrava que nos dados de transcriptoma de $T$. diversipes, ainda no mestrado, havia transcritos "contaminantes" de Wolbachia. Geralmente, dados gerados para montagem de genomas podem ser analisados sob outras perspectivas. Aproveitamos estes dados para montar o genoma de Wolbachia. Ao mesmo tempo fizemos uma prospecção da presença deste endossimbionte em hospedeiros de outras populações. Estes resultados foram apresentados como Apêndice I nesta tese. Nós mostramos que todos os indivíduos analisados foram infectados por duas linhagens diferentes de Wolbachia, ambas do supergrupo A. Devido a esta distribuição uniforme, inferimos que não há uma relação entre o padrão de distribuição de Wolbachia e a estrutura populacional descrita para as fêmeas de T. diversipes. Assim como não há relação entre a presença da bactéria e o viés sexual descrito para 
algumas das populações. Fica em aberto a busca pelo entendimento dos efeitos de Wolbachia em T. diversipes.

Esta tese traz grandes contribuições para a área da diapausa, principalmente envolvendo o grupo das abelhas. Apresentamos mecanismos moleculares da diapausa em T. divesipes que são conservados comparados com outras espécies de insetos e mostramos que há assinaturas genômicas específicas à fase em que a diapausa ocorre (desenvolvimento ou adulta). Além disso,

apresentamos novas perspectivas sobre a relação da diapausa com a evolução do comportamento social e sobre a relação de $T$. diversipes com seu endossimbionte.

\section{Referências}

Alves-dos-Santos I, Melo GAR, Rozen JR JG (2002) Biology and immature stages of the bee tribe Tetrapediini (Hymenoptera: Apidae). Am Museum Novit 3377:1-45

Hunt JH, Amdam GV (2005) Bivoltinism as an antecedent to eusociality in the paper wasp genus Polistes. Science (80- ) 308:264-267. doi: 10.1126/science.1109724

Santos PKF, Araujo NS, Françoso E, et al (2018) Diapause in a tropical oil-collecting bee: Molecular basis unveiled by RNA-Seq. BMC Genomics 19:1-11. doi: 10.1186/s12864-0184694-X

Santos PKF, Arias MC, Kapheim KM (2019) Loss of developmental diapause as a prerequisite for social evolution in bees. Biol Lett 15:1-7. doi: 10.1101/649897 